\title{
Psychosocial mechanisms of the pain and quality of life relationship for chronic prostatitis/chronic pelvic pain syndrome (CP/CPPS)
}

\author{
Adrijana Krsmanovic, MSc; ${ }^{*}$ Dean A. Tripp, PhD; ; J. Curtis Nickel, MD, FRCSC,; Daniel A. Shoskes, MD;* \\ Michel Pontari, MD;: Mark S. Litwin, MD, MPH;: Mary F. McNaughton-Collins, MD
}

*Psychology, Queen's University, Kingston, ON; †Psychology, Anesthesiology and Urology, Queen's University, Kingston, ON; §Urology, Queen's University, Kingston, ON; *Urology, Glickman Urological and Kidney Institute, Cleveland, Ohio; EUrology, Temple University, Philadelphia, PA; EUrology, David Geffen School of Medicine at UCLA, Los Angeles, California and Health Policy and Management, UCLA Fielding School of Public Health, Los Angeles, California; "Massachusetts General Hospital, Boston, Massachusetts

Cite as: Can Urol Assoc J 2014;8(11-12):403-8. http://dx.doi.org/10.5489/cuaj.2179 Published online December 15, 2014.

\section{Abstract}

Introduction: Chronic prostatitis/chronic pelvic pain syndrome (CP/CPPS) is a prevalent, chronic pelvic pain condition largely unresponsive to medical interventions. Psychosocial risk factors are associated with poor outcomes in CP/CPPS, but have not been examined for their intervening roles between pain and reduced quality of life (QoL). This study aimed to determine if psychosocial risk factors (i.e., patient coping and catastrophizing) mediate the association between pain and QoL.

Methods: Using a cross sectional design, 175 men with CP/CPPS (mean age 46.83; SD 10.86) were recruited from tertiary care urology clinics and completed questionnaires on demographics, pain, QoL, pain coping, depression, and catastrophizing. An exploratory factor analysis was conducted and aggregate factor scores were examined to improve the amount of meaningful measurement to be used in multiple mediations. The models specified multiple risk factors as mechanisms between pain and both physical and mental QoL as the primary outcome measurements.

Results: Four aggregate psychosocial factor scores were produced from the psychosocial measures (i.e., illness and wellness-focused behavioural coping, depression and catastrophizing). Illnessfocused coping partially mediated the relationship between pain and physical QoL. However, catastrophizing and illness-focused coping fully mediated the relation between pain and mental QoL, showing the association between pain and mental QoL was no longer significant when catastrophizing and illness-focused coping were in the model.

Conclusion: Psychosocial factors function as mechanisms between higher pain and they are associated diminished mental QoL. These results introduce illness-focused coping as an important biopsychosocial target in CP/CPPS management.

\section{Introduction}

Chronic prostatitis/chronic pelvic pain syndrome (CP/CPPS) is a male urologic condition characterized by pain in the perineum, pelvic area, and/or genitalia. ${ }^{1}$ It is commonly associated with urinary frequency, dysuria, and incomplete voiding. ${ }^{2}$ Prostatitis is a common genitourinary diagnosis for men under $50,{ }^{3}$ with point prevalence rates estimated at $2 \%$ to $10 \%$, with some as high as $15 \%$ to $16 \%$ in Asian, European and North American samples. ${ }^{4}$ The international prevalence in adolescents ranges from $8 \%$ in North Americans ${ }^{5}$ to $13 \%$ in Africans. ${ }^{6}$ Most medical treatments are considered largely ineffective, ${ }^{2}$ and patients report diminished quality of life (QoL), alongside stable reports of pain, disability, depression, anxiety, and catastrophizing over a 2-year period.? Due to the current "non-cure" status of many men suffering from CP/CPPS and the high rate of psychological distress manifest in this syndrome, effective biopsychosocial management for QoL is a clear mandate.

CP/CPPS QoL is comparable to that in patients with myocardial infarct, angina, or Crohn's disease, ${ }^{8}$ and worse than that of those with diabetes mellitus or congestive heart failure. ${ }^{9} \mathrm{CP} / \mathrm{CPPS}$ pain is associated with diminished QoL even when controlling for age, urinary symptoms, depressive symptoms, and partner status. ${ }^{10}$ Although studies show that psychosocial factors can predict diminished CP/CPPS QoL, specified mechanisms of these observed relations have not been examined. The biopsychosocial experience of patients with CP/CPPS is critical for improved patient well-being, and a deeper understanding of the negative pain-QoL relationship is needed to advance patient management. ${ }^{10}$

The urology and pain literature indicates that mood, cognitive appraisals (i.e., catastrophizing), and behavioural pain coping strategies are predictors for pain and QoL. Depression is elevated in CP/CPPS, ${ }^{11}$ and catastrophizing is associated with greater pain in CP/CPPS, ${ }^{12}$ as well as female chronic pelvic pain. ${ }^{13}$ Behavioural coping for pain is also important, usually expressed in the form of wellness-focused and illness-focused coping. Illness-focused coping, such as 
Krsmanovic et al.

guarding, resting, and asking for assistance, is associated with greater pain and poorer QoL, ${ }^{14}$ while pain-contingent rest specifically is associated with greater CP/CPPS painassociated disability. ${ }^{12}$ In contrast, wellness-focused coping (relaxation, exercise, coping self-statements, task persistence) is associated with lower pain, ${ }^{15}$ lower depression, and better $\mathrm{QoL}^{16}$ in chronic pain patients. No CP/CPPS research has examined the full spectrum of behavioural coping strategies.

Although psychosocial variables are associated with $\mathrm{CP} /$ CPPS pain and QoL, ${ }^{12,14}$ the mediating or "mechanistic" role of such variables remains unexamined. This study's aim was to identify which pain appraisals and/or behavioural coping strategies might act as mediators for pain and QoL in CP/CPPS.

\section{Methods}

\section{Participants}

We included 175 men diagnosed with CP/CPPS by urologists at tertiary care clinical centres, specifically those who had symptoms of pain in the pelvic region for at least 3 of the last 6 months. The participants were all enrolled in the National Institutes of Health Chronic Prostatitis Cohort Study, which has been described previously. ${ }^{17}$

Demographic questions included age, education, ethnicity and employment status.

\section{Medical symptoms}

The National Institutes of Health Chronic Prostatitis Symptom Index (NIH-CPSI) uses 9 items to assess CP/CPPS symptoms, examining pain/discomfort, urinary symptoms, and QoL. Responses were binary (yes/no) and Likert-type. ${ }^{18}$

The Short-Form McGill Pain Questionnaire (SF-MPQ) measures self-reported current pain. Patients specified their pain intensity by indicating the degree to which each of the 15 descriptors described their pain, on a 4-point scale from 0-3 "None-Severe."19

The Center for Epidemiologic Studies Depression Scale (CES-D) assesses self-reported depressive symptomatology using 20 items. Participants indicated how often they have associated with a statement over the past week, on a 4-point scale ranging from 0-3 "Rarely or none of the time $(<1$ day)Most or all of the time (5-7 days)." 20

The Pain Catastrophizing Scale assesses cognitive appraisals of the pain experience. The scale is summed for a total score with 3 subscales (rumination, magnification and helplessness) using 13 items. Patients indicate the degree to which they catastrophize when in pain, ranging from 0-4 "Not at all-All the time."21

The Chronic Pain Coping Inventory assesses chronic pain coping strategies using 8 subscales comprised of 64 items.
Each subscale assesses different coping: Illness-Focused Coping, Wellness-Focused Coping, and Other Coping. Items are scored from 0 to 7 , indicating number of days a strategy was used. ${ }^{22}$

The Medical Outcomes Short Form-12 Item Health Survey (SF-12) consists of 12 items with two subscales: Physical and Mental Component Summary. ${ }^{23}$

\section{Procedure}

Following institutional approval, patients were informed about the study individually. Patients agreeing to participate received a questionnaire package and a pre-stamped study centre addressed envelope. Patients who returned the package with a signed consent were included in the study.

\section{Data analysis}

Data analysis was conducted using Statistical Packages for Social Sciences (SPSS) version 21. The data were inspected for normality, multicollinearity, and missing data. Participants missing $>20 \%$ of a measure were removed from the dataset, and the remaining data missing less than $20 \%$ of the items on any measure underwent a missing value replacement with the item mean of the sample. ${ }^{24}$ About $15 \%$ of participants had items replaced due to missing data, which was randomly distributed across measures. To reduce the psychosocial measures to parsimonious and meaningful measurements, we examined the factor structure of the pain appraisal and behavioural coping measures in aggregate with exploratory factor analysis. A principal axis extraction and direct oblimin rotational technique were used, allowing for correlations between factors. ${ }^{25}$ Factor composition criteria included parallel and scree plot analysis, interpretability, and replicability. Factor loadings $>0.3$ were considered substantial, while loadings $<0.3$ were dropped from analyses. The empirical factors were then used in multiple mediation models for pain and QoL (physical and mental). Models followed standard guidelines. ${ }^{26}$

\section{Results}

We tallied demographic characteristics of the sample (Table 1 ) and the means, standard deviations and ranges for the subscales of the individual measures (Table 2).

Factor analysis was conducted for appraisal and coping using their 15 subscales. Kaiser-Meyer-Olkin measure of sampling adequacy was above the recommended value and Bartlett's test of sphericity was significant, $\chi^{2}(120)=1210$, $p<0.01)$, indicating the analysis was appropriate. Various criteria for extracting factors were examined and 4 factors were selected and extracted. Aggregate factor scores were computed and specified in subsequent analyses as illness- 


\begin{tabular}{lc}
\hline Table 1. Sample demographics & \\
\hline Age (years \pm SD) & $\mathbf{4 6 . 8 3} \pm \mathbf{1 0 . 8 6}$ \\
\hline Education & \\
Less than high school & $9.7 \%$ \\
High school or GED & $52.6 \%$ \\
Some college or university & $34.9 \%$ \\
Graduate/professional school & $2.9 \%$ \\
Ethnicity & \\
White/Caucasian & $86.9 \%$ \\
Black/African-American & $5.1 \%$ \\
Spanish/Hispanic/Latino & $2.3 \%$ \\
Asian & $1.7 \%$ \\
Aboriginal & $0.6 \%$ \\
Other & $3.4 \%$ \\
Employment Status & \\
Employed & $80.0 \%$ \\
Retired & $12.6 \%$ \\
On disability & $4.0 \%$ \\
Unemployed & $3.4 \%$ \\
\hline SD: standard deviation; GED: General Educational Development. \\
\hline
\end{tabular}

focused coping, wellness-focused coping, catastrophizing, and depression.

\section{Physical QoL}

Four psychosocial risk factors (illness- and wellness-focused coping, catastrophizing, and depression) were proposed to mediate the relationship between pain and physical QoL (Fig. 1). The associations between pain and the psychosocial factors were positive, indicating that patients who experience more pain tend to use more behavioural coping strategies, whether they are illness-focused or wellness-focused, have greater catastrophic thinking, and experience more depression. Moreover, the association between the illnessfocused coping and physical QoL was negative, indicating that patients who use these types of coping strategies more frequently tend to have poorer physical QoL. The associations between depression, wellness- focused coping, and catastrophizing with physical QoL were not significant. The group effect of the 4 factors produced a significant partial mediation effect of the pain and physical QoL relationship (i.e., the total effect of pain on physical QoL was significant, and remained significant after inclusion of proposed mediators). In examining specific indirect effects of the mediators, only illness-focused coping was significant $(Z=-2.78$, $p<0.01)$.

\section{Mental QoL}

Due to associations, only illness-focused coping and catastrophizing were proposed mediators of the relationship between pain and mental QoL. Depression was not included because of its high association with mental QoL $(r=-0.76$,

\begin{tabular}{|c|c|c|c|}
\hline Measure & Mean & SD & Range \\
\hline CPSI - Total symptom score & 16.47 & 8.67 & $0-39.00$ \\
\hline MPQ - Pain total score & 7.51 & 7.35 & $0-30.00$ \\
\hline CES-D - Total score & 16.52 & 6.81 & $0-36.00$ \\
\hline PCS - Total & 16.30 & 9.52 & $0-48.00$ \\
\hline $\mathrm{CPCl}$ - Asking for assistance & 0.52 & 0.91 & $0-4.50$ \\
\hline $\mathrm{CPCl}-$ Coping self-statements & 1.63 & 1.62 & $0-6.55$ \\
\hline $\mathrm{CPCl}$ - Exercise/stretch & 1.57 & 1.72 & $0-7.00$ \\
\hline $\mathrm{CPCl}$ - Guarding & 0.99 & 1.34 & $0-5.78$ \\
\hline $\mathrm{CPCl}$ - Relaxation & 0.90 & 0.97 & $0-4.00$ \\
\hline $\mathrm{CPCl}$ - Resting & 1.69 & 1.65 & $0-6.43$ \\
\hline CPCI - Social support & 0.73 & 1.06 & $0-6.00$ \\
\hline $\mathrm{CPCl}$ - Task persistence & 4.01 & 2.15 & $0-7.00$ \\
\hline SF12 - Physical QoL & 47.76 & 9.50 & $15.26-63.46$ \\
\hline SF12 - Mental QoL & 46.68 & 10.00 & $21.05-65.29$ \\
\hline \multicolumn{4}{|c|}{$\begin{array}{l}\text { CPSI: Chronic Prostatitis Symptom Index; MPQ: McGill Pain Questionnaire; CES-D: Center } \\
\text { for Epidemiologic Studies Depression Scale; PCS: Pain Catastrophizing Scale; CPCI: Chronic } \\
\text { Pain Coping Inventory; SF12: Medical Outcomes Short Form-12 Item Health Survey; QoL: } \\
\text { Quality of Life. }\end{array}$} \\
\hline
\end{tabular}

$p<0.01$ ), as previously reported..$^{13}$ Wellness-focused coping was excluded from the model due to its non-significant correlation with the dependent variable $(r=-0.09, p=0.24)$. The associations between psychosocial mediators and mental QoL were negative, indicating that patients with greater catastrophic thinking and more frequent use of illnessfocused coping tend to have lower mental QoL (Fig. 2). The group effect of illness-focused coping and catastrophizing produced a full mediation effect of the pain and mental QoL relationship (i.e., the total effect of pain on mental QoL is not significant once psychosocial mediators are included in the model). In examining specific indirect effects of the proposed mediators, both illness-focused coping $(Z=-3.83$, $p<0.01)$ and catastrophizing $(Z=-2.29, p<0.01)$ emerged as significant mediators.

\section{Discussion}

This is the first study to show how empirically derived biopsychosocial variables may explain the pain-QoL relationship manifest in CP/CPPS using multiple mediation modelling. Illness-focused coping was a significant mediator in the observed relationship for the pain/physical QoL model, while illness-focused coping and catastrophizing were significant in the pain/mental QoL model.

These findings show several associated mechanisms are important in explaining the CP/CPPS pain and QoL relationships. In the mental QoL model, the catastrophizing mediation suggests that in men suffering from CP/CPPS, symptoms may become disabling through cognitive mechanisms, where catastrophizing creates an inability to disengage from 
Krsmanovic et al.

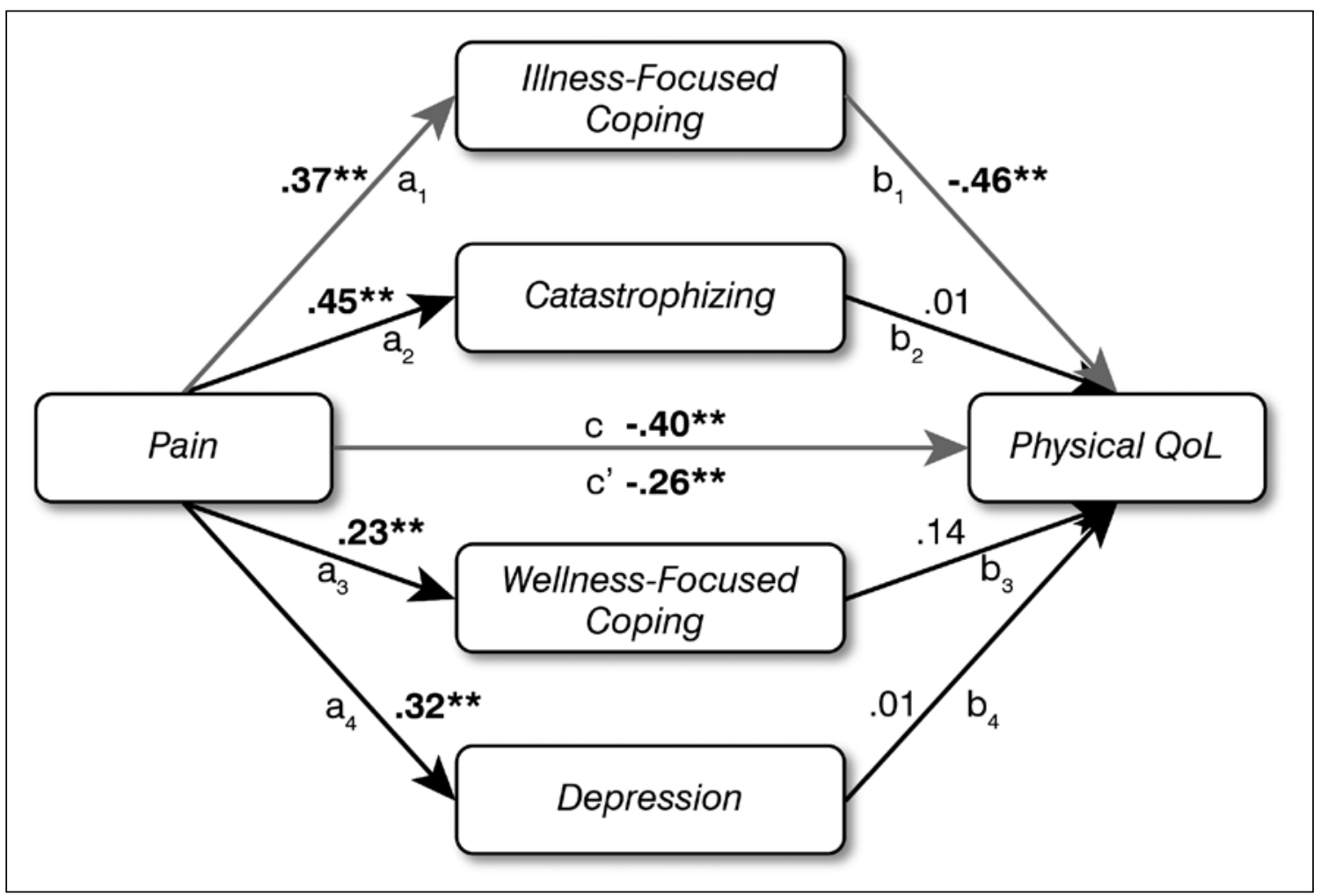

Fig. 1. Multiple Mediation Model for Physical Quality of Life. Standardized regression coefficients $(\beta)$ for the relationship between pain and physical quality of life, as mediated by psychosocial variables. ${ }^{* *} p<0.01$.

negative pain ruminations. ${ }^{27}$ This previously-identified style of negative CP/CPPS processing $5,7,10,12,14$ can be modified, and reductions in catastrophizing have been shown to be associated with reductions in CP/CPPS symptoms. ${ }^{28}$

This study also strongly indicated that illness-focused coping is an important target in improving patients' mental and physical QoL. Previous research has examined the use of pain-contingent rest as a coping strategy in men with $\mathrm{CP} /$
CPPS, and found that it predicted disability ${ }^{12}$ and poorer physical QoL. ${ }^{14}$ However, no studies to date have made use of other illness-focused coping strategies in CP/CPPS samples. In other chronic pain samples, individuals reporting illness-focused coping, reported not only decreased QoL, ${ }^{16}$ but also more pain and depressive symptoms. ${ }^{29}$ Supporting previous research, illness-focused coping was associated with pain and depression in this study.

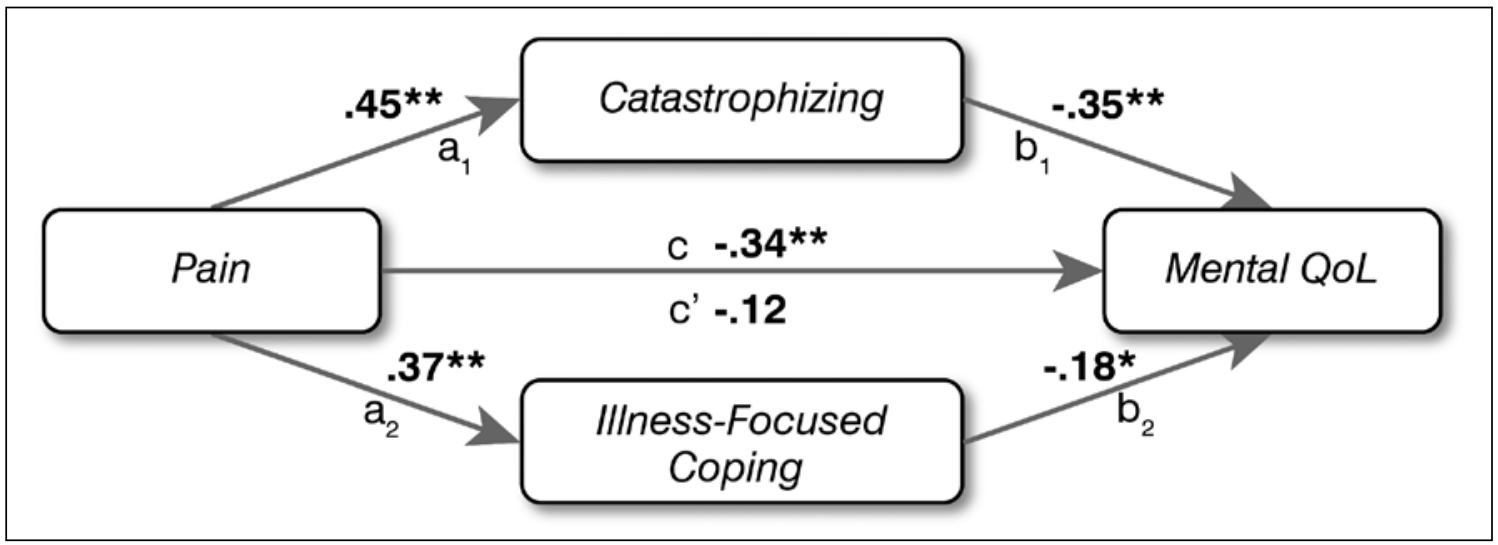

Fig. 2. Multiple Mediation Model for Mental Quality of Life. Standardized regression coefficients $(\beta)$ for the relationship between pain and mental quality of life, as mediated by psychosocial variables. ${ }^{*} p<0.05,{ }^{* *} p<0.01$. 


\section{Clinical implications}

These novel findings underscore specific cognitive and behavioural coping factors as detrimental to CP/CPPS QoL. To improve mental QoL, catastrophizing should be reduced. Interventions have been successful in reducing catastrophizing in CP/CPPS ${ }^{28}$ by teaching patients how to identify and modify catastrophic interpretations of symptom-associated life events, and by promoting renewed social and physical activity engagement. Moreover, physicians can address patients' catastrophizing in their offices using various strategies (e.g., increasing patient self-awareness, suggesting readings for self-management). ${ }^{30}$ Likewise, illness-focused coping, the other significant mechanism between pain and mental/physical QoL, can also be altered through discussions with patients, ${ }^{30}$ specifically examining the adoption of a sedentary lifestyle due to symptoms. Pain coping, such as guarding, pain-contingent rest and asking for assistance, can signal a sense of helplessness linked to inactivity. It is important to stress wellness and activity at the highest attainable level, as well to examine the appropriate use of social support. Illness-focused coping also has broader implications for social functioning, because pain patients reporting illness-focused coping tend to also report less work, school and social activity, and use more healthcare services. ${ }^{29}$

\section{Study limitations/future research}

Although the cross-sectional design limits causality of findings, these results were consistent with other pain and QoL research. ${ }^{12,14}$ The generalizability of these findings may be considered limited due to the sample characteristics. However, recent studies of CP-like symptoms from Africa and Canada found that both point estimates and the impact of symptom indices and depression on QoL were similar to this study. ${ }^{6,13}$ Finally, while patients were drawn from multiple locations, all centres were specialty tertiary referral clinics and as such this sample might not reflect characteristics of patients in primary urologic care.

Given that this study is novel, future research should examine these relations using a larger cohort. In addition, while psychosocial risk factors negatively impact QoL in $\mathrm{CP} / \mathrm{CPPS}$, other variables, including the duration and type of medical treatment of patients, should be examined to account for how medical treatments may be contributing to psychological outcomes.

\section{Conclusion}

This study contributes to the CP/CPPS literature by identifying the functions of key psychosocial risk factors that promote diminished QoL. Catastrophizing and illness-focused coping fully mediated the relationship between pain and mental QoL, while illness-focused coping partially mediated pain and physical QoL. These results require the CP/ CPPS biopsychosocial treatment model to highlight and seek resolution of such QoL associated factors.

Competing interests: Adrijana Krsmanovic, Dr. Tripp, Dr. Shoskes, Dr. Pontari, Dr. Litwin and Dr. McNaughton-Collins declare no competing financial or personal interests. Dr. Nickel is a consultant for Glaxo-Smith-Kline, Taris Biomedical, Pfizer, Eli Lilly, Johnson\&Johnson, Farr Labs, Astellas, Trillium Therapeutics, Auxillium, Ferring and Tribute. He has also participated in clinical trials with Glaxo-Smith-Kline, Taris Biomedical, Pfizer and Eli Lilly.

This paper has been peer-reviewed.

\section{References}

1. Krieger JN, Egan KJ, Ross SO, et al. Chronic pelvic pains represent the most prominent urogenital symptoms of "chronic prostatitis." J Urol 1996;48:715-22. http://dx.doi.org/10.1016/S00904295(96)00421-9

2. Habermacher GM, Chason JT, Schaeffer AJ. Prostatitis/chronic pelvic pain syndrome. Annu Rev Med 2006;57:195-206. http://dx.doi.org/10.1146/annurev.med.57.011205.135654

3. McNaughton-Collins M, Stafford RS, O'Leary MP, et al. How common is prostatitis? A national survey of physician visits. J Urol 1998;159:1224-8. http://dx.doi.org/10.1016/S0022-5347(01)63564-X

4. Krieger JN, Riley DE, Cheah PY, et al. Epidemiology of prostatitis: New evidence for a world-wide problem. World J Urol 2003;21:70-4. http://dx.doi.org/10.1007/s00345-003-0329-0

5. Tripp DA, Nickel JC, Ross S, et al. Prevalence, symptom impact and predictors of chronic prostatitis-like symptoms in Canadian males aged 16-19 years. BJU Int 2008;103:1080-4

6. Tripp D, Nickel J, Pikard J, et al. Chronic prostatitis-like symptoms in African males aged 16-19 years. Can J Urol 2012; 19:6081-7.

7. Tripp DA, Nickel JC, Shoskes D, et al. A 2-year follow-up of quality of life, pain, and psychosocial factors in patients with chronic prostatitis/chronic pelvic pain syndrome and their spouses. World I Urol 2013;31:733-9. http://dx.doi.org/10.1007/s00345-013-1067-6

8. Wenninger $K$, Heiman JR, Rothman I, et al. Sickness impact of chronic nonbacterial prostatitis and its correlates. J Urol 1996;155:965-8. http://dx.doi.org/10.1016/S0022-5347(01)66359-6

9. McNaughton-Collins M, Pontari MA, $0^{\prime}$ Leary MP, et al. Quality of life is impaired in men with chronic prostatitis: Results from the NIH Cohort Study. JGIM 2001;16:656-62. http://dx.doi.org/10.1111/ j.1525-1497.2001.01223.x

10. Tripp D, Nickel J, Landis J, et al. Predictors of quality of life and pain in chronic prostatitis/chronic pelvic pain syndrome: Findings from the National Institutes of Health Chronic Prostatitis Cohort Study. BJU Int 2004;94:1279-82.

11. Clemens JQ, Brown SO, Calhoun EA. Mental health diagnoses in patients with interstitial cystitis/painful bladder syndrome and chronic prostatitis/chronic pelvic pain syndrome: A case/control study. J Urol 2008;180:1378-82. http://dx.doi.org/10.1016/i.juro.2008.06.032

12. Tripp DA, Nickel JC, Wang Y, et al. Catastrophizing and pain-contingent rest predict patient adjustment in men with chronic prostatitis/chronic pelvic pain syndrome. Pain 2006;7:697-708. http://dx.doi. org/10.1016/i.pain.2006.03.006

13. Tripp D, Nickel IC, Fitzgerald MP, et al. Sexual functioning, catastrophizing, depression, and pain, as predictors of quality of life in women with interstitial cysitis/painful bladder syndrome. J Urol 2009;73:987-92. http://dx.doi.org/10.1016/i.urology.2008.11.049

14. Nickel JC, Tripp DA, Chuai S, et al. Psychosocial variables affect the quality of life of men diagnosed with chronic prostatitis/chronic pelvic pain syndrome. BJU Int 2007;101:59-64.

15. Hadjistavropoulos HD, Macleod FK, Asmundson GJG. Validation of the chronic pain coping inventory. Pain 1999;80:471-81. http://dx.doi.org/10.1016/S0304-3959(98)00224-3

16. Garcia-Campayo J, Pascual A, Alda M, et al. Coping with fibromyalgia: Usefulness of the Chronic Pain Coping Inventory-42. Pain 2007;132:S68-76. http://dx.doi.org/10.1016/i.pain.2007.02.013

17. Schaeffer AJ, Landis JR, Knauss JS, et al. Demographic and clinical characteristics of men with chronic prostatitis: The National Institutes of Health Chronic Prostatitis Cohort (CPC) Study. J Urol 2002; 168:5938. http://dx.doi.org/10.1016/S0022-5347(05)64686-1 
Krsmanovic et al.

18. Litwin MS, McNaughton-Collins M, Fowler FJ, et al. The National Institutes of Health chronic prostatitis symptom index: Development and validation of a new outcome measure. J Urol 1999;162:369-75. http://dx.doi.org/10.1016/S0022-5347(05)68562-X

19. Melzack R. The short-form McGill Pain Questionnaire. Pain 1987;30:191-7. http://dx.doi. org/10.1016/0304-3959(87)91074-8

20. Radloff LS. The CES-D scale: A self-report depression scale for research in the general population. Appl Psych Meas 1977;1:385.

21. Sullivan MIL, Bishop SR, Pivik J. The pain catastrophizing scale: Development and validation. Psychological Assessment 1995;7:524-32. http://dx.doi.org/10.1037/1040-3590.7.4.524

22. Jensen MP, Turner JA, Romano JM, et al. The chronic pain coping inventory: Development and preliminary validation. Pain 1995;60:203-16. http://dx.doi.org/10.1016/0304-3959(94)00118-X

23. Ware JE, Kosinski M, Keller S. A 12-Item Short-Form Health Survey: Construction of scales and preliminary tests of reliability and validity. Med Care 1996;34:220-33. http://dx.doi.org/10.1097/00005650199603000-00003

24. Tabachnick BG, Fidell LS. Using multivariate statistics. 4th edition. Boston, MA: Pearson Allyn \& Bacon; 2000.

25. Costello AB, Osborne JW. Best practices in exploratory factor analysis: Four recommendations for getting the most from your analysis. Practical Assessment, Research and Evaluation 2005;10(7). http://pareonline. net/pdf/v10n7.pdf. Accessed November 26, 2014.
26. Preacher KJ, Hayes AF. Asymptotic and resampling strategies for assessing and comparing indirect effects in multiple mediator models. Behavior research methods 2008;40:879-91. http://dx.doi.org/10.3758/ BRM.40.3.879

27. Quartana PJ, Campbell CM, Edwards RR. Pain catastrophizing: A critical review. Expert Rev Neurother 2009;9:745-58. http://dx.doi.org/10.1586/ern.09.34

28. Tripp DA, Nickel JC, Katz L. A feasibility trial of a cognitive-behavioural symptom management program for chronic pelvic pain for men with refractory chronic prostatitis/chronic pelvic pain syndrome. Can Urol Assoc J 2011;5:328-32. http://dx.doi.org/ 10.5489/cuaj.10201

29. Gil KM, Carson JW, Sedway JA, et al. Follow-up of coping skills training in adults with sickle cell disease: Analysis of daily pain and coping practice diaries. Health Psychol 2000;19:85-90. http://dx.doi. org/10.1037/0278-6133.19.1.85

30. Tripp DA, Nickel JC. The psychology of urological chronic pelvic pain: A primer for urologists who want to know how to better manage chronic prostatitis and interstitial cystitis. In: AUA Update Series, 2011. Lesson 40, Volume 30. American Urological Association: Linthicum, MD.

Correspondence: Adrijana Krsmanovic, Queen's University, 62 Arch Street, Kingston, 0N K7L 3N6; 0ak93@queensu.ca 